\title{
ON THE REGULAR BEHAVIOUR OF ORTHOGONAL POLYNOMIALS
}

\author{
$B y$ J. L. ULLMAN† \\ [Received 14 July 1970—Revised I June 1971]
}

\section{Introduction}

For a large class of polynomials orthogonal on $I=[-1,1]$ with respect to a non-negative integrable weight function, the zeros have the same limiting distribution. In fact, it has been shown ([1]) that, if $p(x)$ is a non-negative integrable function defined on $I$ and $p(x)>0$ except for a set of measure zero, and $\left\{P_{n}(x)\right\}(n=0,1, \ldots)$ are the associated orthogonal polynomials, then if $-1 \leqq \alpha<\beta \leqq 1$

$$
\lim _{n \rightarrow \infty} \frac{\nu_{n}^{*}(\alpha, \beta)}{n}=\frac{1}{\pi} \int_{\alpha}^{\beta} \frac{d x}{\sqrt{\left(1-x^{2}\right)}},
$$

where $\nu_{n}^{*}(\alpha, \beta)$ is the number of zeros of $P_{n}(x)$ on the interval $[\alpha, \beta]$. (The reference to measure in the previous sentence and in what follows means linear Lebesgue measure, unless otherwise stated. Also meas $E$ or meas $\left(E \backslash E^{\prime}\right)$, for example, will be used to denote the measure of the set $E$ and of $E \backslash E^{\prime}$, respectively.) We shall call this limiting behaviour of the zeros regular behaviour and the associated weight function a regular weight function. In this paper we explore several questions, some suggested by this theorem. We now continue the introduction in a more formal way, beginning with a brief recapitulation. Where references are not given, proofs of the lemmas and theorems will be found in $\$ \S 3-6$ according to a plan given in $\S 2$. Section 7 discusses some unsolved problems.

Definition 1.1. Let $I$ denote the interval $[-1,1]$. Let $M(I)$ be the class of measurable subsets of $I$ having positive measure. For $E \in M(I)$, let $P(E)$ be the class of non-negative integrable functions having the property that, if $p(x) \in P(E)$, then $\mathrm{S}(p(x))$, the support of $p(x)$ (defined as $\{x: x \in I, p(x)>0\})$, satisfies $\mathrm{S}(p(x))=E$. We finally let

$$
P=\bigcup_{E \in M(I)} P(E) \text {. }
$$

Lemma 1.1 ([8] p. 24). For $p(x) \in P$, there is a unique set of polynomials $\left\{P_{n}(x)\right\}$, also written $\left\{P_{n}(x \mid p)\right\}, P_{n}(x)=x^{n}+\ldots(n=0,1, \ldots)$, and a unique

$\dagger$ The author gratefully acknowledges the support of National Science Foundation Grant GP.8934, and the valuable comments of the Referee. 
set of positive constants $\left\{N_{n}(p)\right\}(n=0,1, \ldots)$ satisfying the conditions

$$
\int_{-1}^{1} P_{m}(x) P_{n}(x) p(x) d x=\delta_{m n}\left(N_{n}(p)\right)^{2} \quad(m, n=0,1, \ldots),
$$

where $\delta_{m n}$ is the Kronecker delta. We call $\left\{P_{n}(x)\right\}$ the set of orthogonal polynomials associated with $p(x)$ and we call $N_{n}(p)$ the norm of $P_{n}(x)$. The zeros of $P_{n}(x)(n=1, \ldots)$ are simple and lie on the interval $I$.

Defrnimion 1.2. For $p(x) \in P$ and $\alpha, \beta$ satisfying $-1 \leqq \alpha<\beta \leqq 1$, let $\nu_{n}^{*}(\alpha, \beta)$ be the number of zeros of $P_{n}(x \mid p)$ lying in $[\alpha, \beta]$. If $(1.1)$ is satisfied we say that the zeros of $\left\{P_{n}(x \mid p)\right\}$ have regular behaviour and that $p(x)$ is a regular weight function.

Defrnition 1.3. Let $N(I)$ be the subclass of $M(I)$ characterized by the property that, if $E \in N(I)$, then, for all $\alpha, \beta$ such that $-1 \leqq \alpha<\beta \leqq 1$,

$$
\operatorname{meas}(\{x: x \in E \cap[\alpha, \beta]\})>0 \text {. }
$$

LEMMa 1.2. A necessary condition for $p(x) \in P$ to be regular is that $\mathrm{S}(p(x)) \in N(I)$.

Defrnition 1.4. A set $E \in N(I)$ is called a determining set if all $p(x) \in P(E)$ are regular weight functions.

We can now recast the theorem of the opening paragraph as follows.

Theorem 1.1 ([1]). If $E \in M(I)$ and meas $E=2$, then $E$ is a determining set.

We note that if meas $E=2$, then $E \in N(I)$, so that the necessary condition for determining sets of Lemma 1.2 is satisfied.

Definition 1.5. For any set $E \subset 1$, by $C(E)$, the capacity of $E$, we shall mean the inner logarithmic capacity (see Definition 3.1). For $E \in M(I)$, by $L(E)$, the lower capacity of $E$, we mean $\inf C\left(E^{\prime}\right)$, where we consider all measurable sets $E^{\prime}$, for which $E^{\prime} \subset E$ and meas $\left(E^{\prime} \backslash E^{\prime}\right)=0$. We refer to such a set $E^{\prime}$ as an equimeasurable subset of $E$. If $L(E)=C(E)$ we say that the capacity of $E$ is stable, and that $E$ has stable capacity $C(E)$.

Let $E \in M(I)$. By Theorem $3.1 C(I)=1 / 2$, and by Definition 3.1 if $E_{i} \in M(I)(i=1,2)$, and, if $E_{1} \subset E_{2}$, then $C\left(E_{1}\right) \leqq C\left(E_{2}\right)$. It thus follows that $L(E) \leqq C(E) \leqq C(I)=1 / 2$. Thus, if $L(E)=1 / 2, E$ has stable capacity $1 / 2$.

Theorem 1.2. Let $E \in N(I) . A$ necessary and sufficient condition that $E$ be a determining set is that $E$ have stable capacity 1/2. When $E \in N(I)$ has stable capacity $1 / 2$, for any $p(x) \in P(E)$ we have $\lim _{n \rightarrow \infty}\left(N_{n}(p)\right)^{1 / n}=1 / 2$. 
LEMma 1.3. (a) The set I has stable capacity 1/2. (b) For any $\varepsilon>0$, there is a set $E, E \in N(I)$, with meas $E<\varepsilon$ and $L(E)=1 / 2$. (c) There are sets $E \in N(I)$ with $L(E)<1 / 2$.

In the above lemma, (a) shows that Theorem 1.2 contains Theorem 1.1, (b) shows that the sufficient condition stated by Theorem 1.1 is not a necessary condition for regularity, and (c) shows that not all sets in $N(I)$ are determining sets.

We now continue our introduction with theorems which describe the possible behaviour of the zeros of orthogonal polynomials and their norms for $p(x) \in P$ and $\mathrm{S}(p(x)) \in N(I)$, but where $\mathrm{S}(p(x))$ need not be a determining set.

Definition 1.6. For any compact set $K, K \subset R, R$ denoting the real axis, let $\Omega(K)$ be the class of unit measures $\mu$ defined on the Borel measurable subsets of $R$ with carrier $c(\mu)$ contained in $K$, where $c(\mu)=\{x: \mu((x-\varepsilon, x+\varepsilon))>0$, for all $\varepsilon>0\}$. Denote $\Omega(I)$ simply as $\Omega$. For $\mu \in \Omega$, let $U(z, \mu)=U(\mu)=\int \log (1 /|z-t|) d \mu$. The integral is defined as a Lebesgue integral with respect to the measure $\mu$ and is known as the potential of $\mu$.

THEOREM 1.3. There is a unique measure $\mu \in \Omega$ such that $U(x, \mu) \geqq \log 2$ for all $x \in I$, with the possible exception of a Borel set of capacity zero. We denote this measure by $\mu_{I}$, and note that

$$
\mu_{I}(B)=\frac{1}{\pi} \int_{B} \frac{d x}{\sqrt{\left(1-x^{2}\right)}}
$$

for all Borel sets $B, B \subset I$.

LEMma 1.4. Let $p(x) \in P$ and let $\left\{P_{n}(x)\right\}$ be the set of associated orthogonal polynomials. For fixed $n \geqq 1$, let $x_{i, n}(i=1, \ldots, n)$ denote the zeros of $P_{n}(x)$.

Let $\nu_{n}$ be the measure, defined on the Borel subsets of $I$, determined by defining $\nu_{n}\left(x_{i, n}\right)=1 / n(i=1, \ldots, n)$ and $\nu_{n}(E)=0$ when $P_{n}(x) \neq 0$ on $E$. Then $p(x)$ is a regular weight function if and only if

$$
\lim _{n \rightarrow \infty} \nu_{n}=\mu_{I} \text {. }
$$

The convergence of measures is defined in Definition 4.1.

Theorem 1.4. Let $E \in N(I)$. Then $L(E)>0$. If a measure $\mu \in \Omega$ exists such that $U(\mu) \geqq \log (1 / L(E))$ for $x \in E$, except possibly for a set of measure zero, then it is unique. When the measure exists, then equality must hold on $B$, an equimeasurable subset of $E$ that is a Borel set for which 
$C(B)=L(E)$. We denote the measure, when it exists, by $\mu_{E}^{*}$. Then $\mu_{E}^{*}=\mu_{I}$ if and only if $L(E)=1 / 2$.

Equality of measures is defined in Definition 4.1.

TheOREM 1.5. Let $E \in N(I)$ and let $p(x) \in P(E)$. Then

$$
L(E) \leqq \lim _{n \rightarrow \infty}\left(N_{n}(p)\right)^{1 / n} \leqq \varlimsup_{n \rightarrow \infty}\left(N_{n}(p)\right)^{1 / n} \leqq 1 / 2 .
$$

THEOREM 1.6. (a) For $E \in N(I)$ there are $p(x) \in P(E)$ and an increasing sequence of integers $\left(k_{n}\right)$ such that

$$
L(E)=\lim _{n \rightarrow \infty}\left(N_{k_{n}}(p)\right)^{1 / k_{n}}
$$

(b) Let $E \in N(I)$. For any $p(x)$ for which there is an increasing sequence of integers $\left(k_{n}\right)$ for which (1.6) holds, we have

$$
\lim _{n \rightarrow \infty} \nu_{k_{n}}=\mu_{E}^{*}
$$

where the measures $\left\{\nu_{n}\right\}$ are defined in Lemma 1.4 and the measure $\mu_{E}^{*}$ is defined in Theorem 1.4 .

At this point we remark that Theorem 1.2 can be deduced from Theorems 1.4, 1.5, and 1.6. These theorems contain extra information, however, concerning zero and norm behaviour for orthogonal polynomials associated with $p(x) \in P$ when $\mathrm{S}(p(x))$ is not a determining set. Still further information valid for all sets $E \in N(I)$, whether they are determining sets or not, is contained in the following theorem.

Theorem 1.7. For any $E \in N(I)$ there is a $p(x) \in P(E)$ which is a regular weight function.

What has been described in Theorems 1.2, 1.5, 1.6, and 1.7, we say, belongs to the theory of the first-order asymptotic behaviour of orthogonal polynomials. This terminology is used to distinguish these results from other results, to be summarized in Theorem 1.8 ([2]), concerning another form for describing asymptotic behaviour of orthogonal ploynomials. This latter form has implications for the first-order theory, but does not subsume it, and will be referred to as belonging to the theory of the second-order asymptotic behaviour of orthogonal polynomials.

LEMMA 1.5. Let $f(z)=\frac{1}{2}\left(z+\sqrt{ }\left(z^{2}-1\right)\right)$ with domain

$$
C_{1}=\{z:|z-1|+|z+1|>2\}
$$

where the branch of $\sqrt{ }\left(z^{2}-1\right)$ in $C_{1}$ is chosen so that $\sqrt{ }\left(z^{2}-1\right) / z$ tends to 1 as $z$ tends to infinity. For $p(x) \in P$, the associated orthogonal polynomials 

$\left\{P_{n}(z)\right\}$ have regular behaviour if and only if, for all $z \in C_{1}$,

$$
\lim _{n \rightarrow \infty}\left|P_{n}(z)\right|^{1 / n}=|f(z)| \text {. }
$$

Theorem 1.8 ([2]). Let $\mu \in \Omega$ and denote its absolutely continuous component by $\mu_{\mathrm{a}}$. Let $p(x)$ be the Radon-Nikodym derivative of $\mu_{\mathrm{a}}$ with respect to linear Lebesgue measure. Assume that $c(\mu)$ is an infinite point set. There is then a unique set of polynomials $\left\{P_{n}(x)\right\}=\left\{P_{n}(x \mid \mu)\right\}, P_{n}(x)=x^{n}+\ldots$ $(n=0,1, \ldots)$ and a unique set of positive constants $\left\{N_{n}(\mu)\right\}(n=0,1, \ldots)$ satisfying the conditions

$$
\int P_{m}(x) P_{n}(x) d \mu=\delta_{m n}\left(N_{n}(\mu)\right)^{2} \quad(m, n=0,1, \ldots) .
$$

We call $\left\{P_{n}(x)\right\}$ the set of orthogonal polynomials associated with $\mu$ and we call $N_{n}(\mu)$ the norm of $P_{n}(x)$.

The following statements are equivalent:

$$
\begin{gathered}
\int_{-1}^{1} \frac{\log p(x)}{\sqrt{\left(1-x^{2}\right)}} d x>-\infty, \\
\lim _{n \rightarrow \infty} \frac{\left|P_{n}(z)\right|}{\left|f^{n}(z)\right|} \text { exists and is not zero for all } z \in C_{1}, \\
\text { for some } \varepsilon>0, \quad 2^{n} N_{n}(\mu) \geqq \varepsilon>0 \quad(n=1, \ldots) .
\end{gathered}
$$

The function $f(z)$ in (1.11) is the one introduced in Lemma 1.5.

The implications for first-order theory are as follows. First of all, if $p(x) \in P$ and (1.10) is satisfied, then we can deduce from (1.11) and Lemma 1.5 that $p(x)$ is regular. It follows from Theorem 1.1, however, that $(1.10)$ is not a necessary condition for regularity.

A further important implication requires a definition.

Definition 1.7. Let $\mu \in \Omega$ and let $c(\mu)$ be an infinite set. If the polynomials $\left\{P_{n}(x \mid \mu)\right\}$ of Theorem 1.8 satisfy (1.8), or if their zeros satisfy (1.1), we say that $\mu$ is a regular measure. If (1.11) is satisfied, we say that $\mu$ is a very regular measure. Likewise, if $p(x) \in P$ satisfies (1.10), we say that it is a very regular weight function.

By Theorem 1.8, we see that whether $\mu$ is very regular or not depends on its absolutely continuous component, in particular on whether or not (1.10) is satisfied. The situation is different for first-order asymptotics.

Theorem 1.9. There is a measure $\mu \in \Omega$, with $c(\mu)$ an infinite set, which is a regular measure and is singular with respect to linear Lebesgue measure.

The measure introduced in this theorem is regular, yet the RadonNikodym derivative of its absolutely continuous component is identically 
zero, and so does not determine a set of orthogonal polynomials and cannot be classified as a regular weight function.

Thus, in first-order theory, the problems of characterizing regular weight functions and regular measures bear a different relationship to each other from that between the corresponding problems for very regular weight functions and very regular measures in the second-order theory.

We conclude this section with a theorem concerning regular measures.

THEOREM 1.10 ([2]). Let $\mu \in \Omega$. Then $\mu$ is a regular measure if

$$
\lim _{n \rightarrow \infty} \omega\left(1 / 2 n^{2}\right)^{1 / n}=1 \text {, }
$$

where

$$
\omega(\delta)=\inf _{x \in I} \mu((x-\delta, x+\delta)) .
$$

\section{Plan of proofs}

Each of $\S \S 3-6$ contains the development of some central technique, and in addition the proofs of the material presented in the introduction that is related to it. In $\S 7$ both general problems and more technical questions that are as yet unsettled are discussed.

Section 3 contains a development of the notions of capacity and stable capacity and the proof of Lemma 1.3. In $\S 4$ the central theme is measures associated with sets, and the section contains the proofs of Theorems 1.3 and 1.4 and Lemmas 1.4 and 1.5. Section 5 is concerned with the connection between norm behaviour and zero distribution and contains proofs for Theorems 1.1, 1.2, 1.5, and 1.6 as well as Lemma 1.2. A sufficient condition that a measure be regular is proved in $\S 6$ as Theorem 1.10 and the proofs of Theorems 1.7 and 1.9 then follow as applications.

\section{Capacity and stable capacity}

We select the energy approach in defining capacity as the most suitable for our applications ([4] p. 280).

Definition 3.1. For any measure $\mu$ defined on the measure space $(R, F)$, where $R$ denotes the real line, and $F$ the $\sigma$-algebra of Borel sets of $R$, let

$$
I(\mu)=\int \log \frac{1}{|z-t|} d(\mu \times \mu),
$$

where $\mu \times \mu$ is the complete product measure defined on $R \times R$. This is called the energy of $\mu$. For $K$ a compact set, $K \subset R$, we let

$$
V(K)=\inf _{\mu \in \Omega(K)} I(\mu),
$$


REGULAR BEHAVIOUR OF ORTHOGONAL POLYNOMIALS 125

where $\Omega(K)$ is defined in Definition 1.6, and call this quantity the equilibrium energy associated with $K$. We then define the capacity of $K$ as $C(K)=\exp (-V(K))$. For any set $E \subset R$, we define the inner capacity of $E$ as $\sup _{K \subset E} C(K)$, where $K$ denotes a compact set. We use the symbol $C(E)$ for inner capacity, and no ambiguity will arise in this paper in referring to $C(E)$ as the capacity of $E$. It is clear that, if $E_{1} \subset E_{2}$, $C\left(E_{1}\right) \leqq C\left(E_{2}\right)$. This is referred to as the monotonicity of capacity.

THEOREM 3.1 ([4] p. 282). (a) Let $K \subset R$ be a compact set of positive capacity. There is a unique measure $\mu_{K}$, where $\mu_{K} \subset \Omega(K)$ and is such that $I\left(\mu_{K}\right)=V(K)$. We refer to $\mu_{K}$ as the Frostman measure of $K$. The potential $U\left(z, \mu_{K}\right)$ (see Definition 1.6) satisfies $U\left(z, \mu_{K}\right) \leqq V(K)$, where equality can hold only on $K$, and may fail to hold there only on a Borel set of type $F_{\sigma}$ of capacity zero.

(b) When $K=I, \mu_{I}$ as defined in this theorem agrees with the measure $\mu_{I}$ defined in (1.3): so there is no conflict of notation. Computation yields $U\left(x, \mu_{I}\right)=\log 2$ for $x \in I, V(I)=\log 2$ and $C(I)=1 / 2$. For $E \subset R$, and $\alpha$ real and non-zero, let $\alpha E=\left\{x: x \alpha^{-1} \in E\right\}$, and $E+\alpha=\{x: x-\alpha \in E\}$, the latter holding even if $\alpha=0$. Then $C(\alpha E)=\alpha C(E)$, and $C(E+\alpha)=C(E)$. Thus, if $L \subset R$ is an interval of length $l, C(L)=l / 4$ and $V(L)=\log (4 / l)$.

Lemma 3.1. (a) ([9] p. 57). If $E_{n} \subset R(n=1, \ldots)$ and $E_{n}$ is a Borel set of capacity zero, then $\bigcup_{n=1}^{\infty} E_{n}$ has capacity zero.

(b) If $E_{n} \subset R(n=1,2)$, and $E_{n}$ is a Borel set, and $E_{2}$ has capacity zero, then

$$
C\left(E_{1} \cup E_{2}\right)=C\left(E_{1} \backslash E_{2}\right)=C\left(E_{1}\right) .
$$

Lemma 3.2. Let $B \subset I$ be a Borel set of type $F_{\sigma}$ and let $B=\bigcup_{n=1}^{\infty} K_{n}$, where $\left\{K_{n}\right\}$ consists of compact sets and $K_{n} \subset K_{n+1}(n=1, \ldots)$. Then $C(B)=\lim _{n \rightarrow \infty} C\left(K_{n}\right)$.

Proof of Lemma 3.2. If $B$ is compact, it may be that, for some integer $N>0, K_{n}=K_{n+1}$ for $n \geqq N$. In this case $B=K_{N}$ and

$$
C(B)=C\left(K_{N}\right)=C\left(K_{N+1}\right)=\ldots \text { : }
$$

so the result is immediate. If there are infinitely many different sets among the $\left\{K_{n}\right\}$ we proceed as follows. By the monotonicity of capacity $C\left(K_{n}\right) \leqq C\left(K_{n+1}\right)(n=1, \ldots)$. Hence $\lim _{n \rightarrow \infty} C\left(K_{n}\right)$ exists, and, again by monotonicity, $\lim _{n \rightarrow \infty} C\left(K_{n}\right) \leqq C(B)$. If for every compact set $K \subset B$

$$
C(K) \leqq \lim _{n \rightarrow \infty} C\left(K_{n}\right)
$$


It would then follow that $C(B) \leqq \lim _{n \rightarrow \infty} C\left(K_{n}\right)$, which would complete the proof. We thus turn to the proof of (3.3).

If $C(B)=0$, for a compact set $K \subset B$, we have $C(K)=0$ by the monotonicity of capacity. Then both sides of the inequality (3.3) are zero, and the inequality is satisfied.

If $C(B)>0$, it is necessary by Lemma 3.1 that we should have $C\left(K_{n}\right)>0$ when $n>N$ for some positive integer $N$. Since we can begin enumeration of $\left\{K_{n}\right\}$ at any point, there is no loss in generality in assuming that $C\left(K_{n}\right)>0(n=1, \ldots)$.

Let $K \subset B$ be a compact set. Since (3.3) is satisfied if $C(K)=0$, we assume that $C(K)>0$. Let $K \cap\left(K_{n} \backslash K_{n-1}\right)=L_{n}$. The sets $\left\{L_{n}\right\}(n=1, \ldots)$, where $K_{0}=\varnothing$, the empty set, are pairwise disjoint Borel sets and $\bigcup_{n=1}^{\infty} L_{n}=K$. The Frostman measure $\mu_{K}$ satisfies

$$
1=\int d \mu_{K}=\sum_{n=1}^{\infty} \int_{L_{n}} d \mu_{K}
$$

Thus, if $\sum_{k=1}^{n} \int_{L_{k}} \mu_{K}=m_{n}, \lim _{n \rightarrow \infty} m_{n}=1$. Thus there is an integer $N>0$ such that, for $n>N, m_{n}>0$. For $n>N$, let $\mu_{K}^{n}$ be the restriction of $\mu_{K}$ to $K_{n}$. Then

$$
V\left(K \cap K_{n}\right) \leqq I\left(\frac{\mu_{K}^{n}}{m_{n}}\right)=\frac{1}{m_{n}{ }^{2}} I\left(\mu_{K}^{n}\right),
$$

where

$$
I\left(\mu_{K}^{n}\right)=\int_{K_{n} \times K_{n}} \log \frac{1}{|z-\bar{t}|} d\left(\mu_{K} \times \mu_{K}\right) .
$$

Since $K \times K=\bigcup_{n=1}^{\infty}\left(K_{n} \times K_{n}\right) \backslash\left(K_{n-1} \times K_{n-1}\right)$, using our convention for $K_{0}$, countable additivity of the integral on the product space yields

$$
\lim _{n \rightarrow \infty} I\left(\mu_{K}^{n}\right)=I\left(\mu_{K}\right) .
$$

Since $I\left(\mu_{K}\right)=V(K)$, it follows from (3.4) and (3.5) that

$$
\varlimsup_{n \rightarrow \infty} V\left(K \cap K_{n}\right) \leqq V(K),
$$

which is equivalent to

$$
\varliminf_{n \rightarrow \infty} C\left(K \cap K_{n}\right) \geqq C(K) .
$$

Finally, by the monotonicity of capacity,

$$
\lim _{n \rightarrow \infty} C\left(K_{n}\right) \geqq \lim _{n \rightarrow \infty} C\left(K \cap K_{n}\right),
$$

so that using (3.6) we obtain (3.3), and the proof is complete. 
Lemma 3.3. Let $E \in M(I)$.

(a) There is an equimeasurable subset of $E$, say $E_{0}$, such that $C\left(E_{0}\right)=L(E)$.

(b) The lower capacity of $E, L(E)$, is positive.

Proof of Lemma 3.3. (a) Since $L(E)=\inf _{E^{\prime}} C\left(E^{\prime}\right)$, where $E^{\prime}$ represents an equimeasurable subset of $E$, there exists a sequence $\left\{E_{n}\right\}(n=1, \ldots)$ of equimeasurable subsets of $E$ such that

$$
L(E)=\lim _{n \rightarrow \infty} C\left(E_{n}\right) .
$$

Now if $E_{0}=\bigcap_{n=1}^{\infty} E_{n}, E_{0}$ is again an equimeasurable subset of $E$, so that $C\left(E_{0}\right) \geqq L(E)$. On the other hand, $C\left(E_{0}\right) \leqq C\left(E_{n}\right)(n=1, \ldots)$, by the monotonicity of capacity, so that by (3.7) $C\left(E_{0}\right) \leqq L(E)$. Thus $C\left(E_{0}\right)=L(E)$.

(b) By Definition 1.1, meas $E=\alpha>0$. Every equimeasurable subset $E^{\prime}$ of $E$ will contain a compact subset $K^{\prime}$ of measure $\geqq \frac{1}{2} \alpha$. By the monotonicity of capacity, $C\left(E^{\prime}\right) \geqq C\left(K^{\prime}\right)$ and $C\left(K^{\prime}\right) \geqq \frac{1}{2} \alpha \cdot \frac{1}{4}=\frac{1}{8} \alpha$ by [9] p. 84. Thus $L(E)$ is positive.

THEOREM 3.2. (a) If $K \subset I$ is a compact set of positive capacity and $\mu_{K}$, its Frostman measure, is absolutely continuous with respect to linear Lebesgue measure, then $K$ has stable capacity.

(b) If $E \subset I$ is a Borel set of type $F_{\sigma}$ with representation $\bigcup_{n=1}^{\infty} K_{n}$, where $K_{n} \subset K_{n+1}(n=1, \ldots)$ and the sets $K_{n}$ are compact and have stable capacity, then $E$ has stable capacity.

Proof of Theorem 3.2. (a) We have to show that, if $K^{\prime}$ is an equimeasurable subset of $K$, then $C\left(K^{\prime}\right)=C(K)$. Since $C\left(K^{\prime}\right) \leqq C(K)$ by the monotonicity of capacity, it remains to show that

$$
C\left(K^{\prime}\right) \geqq C(K) \text {. }
$$

Now $K^{\prime}$ contains an equimeasurable subset $K^{\prime \prime}$ that is a Borel set of type $F_{\sigma}$, having the representation $K^{\prime \prime}=\bigcup_{n=1}^{\infty} K_{n}$, where the $K_{n}$ are compact sets and $K_{n} \subset K_{n+1}(n=1, \ldots)$. By Lemma 3.2

$$
C\left(K^{\prime \prime}\right)=\lim _{n \rightarrow \infty} C\left(K_{n}\right) \text {. }
$$

Now $\mu_{K}(B)=\int_{B} r(x) d x$, where $B \subset I$ is a Borel set and $r(x)$ is the RadonNikodym derivative of $\mu_{K}$ with respect to linear Lebesgue measure. Also

$$
1=\int_{K} r(x) d x=\int_{K^{n}} r(x) d x=\lim _{n \rightarrow \infty} \int_{K_{n}} r(x) d x,
$$

the last step using the countable additivity of the integral. Thus if $m_{n}=\int_{K_{n}} r(x) d x, \lim _{n \rightarrow \infty} m_{n}=1$. Thus there is a positive integer $N$ such that, 
for $n>N, m_{n}>0$. If $\mu_{K}^{n}$ is the restriction of $\mu_{K}$ to $K_{n}$, then, for $n>N$,

$$
V\left(K_{n}\right) \leqq I\left(\frac{\mu_{K}^{n}}{m_{n}}\right)=\frac{1}{m_{n}{ }^{2}} \int_{K_{n} \times K_{n}} \log \frac{1}{|z-t|} r(z) r(t) d z d t .
$$

From (3.10) we obtain

$$
\begin{aligned}
\varlimsup_{n \rightarrow \infty} V\left(K_{n}\right) & \leqq \int_{K^{*} \times K^{*}} \log \frac{1}{|z-t|} r(z) r(t) d z d t \\
& =\int_{K \times K} \log \frac{1}{|z-t|} r(z) r(t) d z d t=V(K),
\end{aligned}
$$

using the countable additivity of the integral on the product space in the first inequality, and the equimeasurability of the subset $K^{\prime \prime} \times K^{\prime \prime}$ of $K \times K$, using Lebesgue measure in the product space, for the second inequality. Thus

$$
\varliminf_{n \rightarrow \infty} C\left(K_{n}\right) \geqq C(K),
$$

and so, using (3.9),

$$
C\left(K^{\prime \prime}\right)=\lim _{n \rightarrow \infty} C\left(K_{n}\right) \geqq C(K) .
$$

By the monotonicity of capacity $C\left(K^{\prime}\right) \geqq C\left(K^{\prime \prime}\right)$, and this fact together with (3.11) yields (3.8).

(b) We note that the sets $\left\{K_{n}\right\}$ are now those introduced in part (b) of the theorem. By Lemma 3.2,

$$
C(E)=\lim _{n \rightarrow \infty} C\left(K_{n}\right)
$$

and we must also show that $C\left(E^{\prime}\right)=C(E)$ for each $E^{\prime}$ which is an equimeasurable subset of $E$. Since $C\left(E^{\prime}\right) \leqq C(E)$ by the monotonicity of capacity, we must show that

$$
C\left(E^{\prime}\right) \geqq C(E) .
$$

If we let $K_{n}^{\prime}=K_{n} \cap E^{\prime}$, then $K_{n}^{\prime}$ is an equimeasurable subset of $K_{n}$ and $\bigcup_{n=1}^{\infty} K_{n}^{\prime}=E^{\prime}$. Thus

$$
C\left(E^{\prime}\right) \geqq C\left(K_{n}^{\prime}\right)=C\left(K_{n}\right),
$$

using first the monotonicity of capacity, and then the stability of the capacity of $K_{n}$. Taking the limit with respect to $n$ in (3.14) and using (3.12), we obtain (3.13), which completes the proof.

Lemma 3.4 ([12] p. 141). Let $K \subset R$ be the union of a finite number of pairwise disjoint compact intervals. Then $\mu_{K}$ is absolutely continuous with respect to linear Lebesgue measure. 
Up to this point we have developed properties of stable capacity. It is now our objective to show the existence, in a constructive manner, of sets $E \in N(I)$ of small measure and stable capacity $1 / 2$.

Lemma 3.5. (a) The function $\cos n \theta(n$ a non-negative integer) is a polynomial of degree $n$ in $\cos \theta$, which we denote by $t_{n}(x), x=\cos \theta$. The polynomial $T_{n}(x)=t_{n}(x) / 2^{n-1}$ is monic. The zeros of $t_{n}(x)$ are given by

$$
x_{k, n}=\cos \frac{2 k-1}{2 n} \pi \quad(k=1, \ldots, n),
$$

and satisfy

$$
\lim _{n \rightarrow \infty}\left(V\left(x_{1, n}, \ldots, x_{n, n}\right)\right)^{1 /(n(n-1)\}}=1 / 2
$$

where

(b) If

$$
V\left(y_{1}, \ldots, y_{n}\right)=\prod_{\substack{i, j=1 \\ i \neq j}}^{n}\left|y_{i}-y_{j}\right|
$$

then

$$
\begin{gathered}
A_{i, j, n}=\left|x_{i, n}-x_{j, n}\right|+\theta_{i, j, n} n^{-3}, \\
\left|\theta_{i, j, n}\right| \leqq 1 \quad(n=1, \ldots ; i, j=1, \ldots, n, i \neq j),
\end{gathered}
$$

$$
\lim _{n \rightarrow \infty}\left(\prod_{\substack{i, j=1 \\ i \neq j}}^{n} A_{i, j, n}\right)^{1 /\{n(n-1)\}}=1 / 2 .
$$

Proof of Lemma 3.5. (a) Since $\cos n \theta=\operatorname{Re}(\cos \theta+i \sin \theta)^{n}$, in the expansion of the right-hand side, $\sin \theta$ enters only to even powers, so that $\cos n \theta$ can be written as a polynomial in $\cos \theta$. The degree of the polynomial in $\cos \theta$, and the leading coefficient are readily seen to be $n$ and $2^{n-1}$ from this representation. Since the $n$ numbers (3.15) are distinct, and

$$
t_{n}\left(\cos \left(\frac{2 k-1}{2 n} \pi\right)\right)=\cos \left(n \frac{2 k-1}{2 n} \pi\right)=0 \quad(k=1, \ldots, n),
$$

they account for all the zeros of $t_{n}(x)$. We next note that

$$
T_{n}(x)=\frac{\cos (n \operatorname{arcos} x)}{2^{n-1}},
$$

so that

$$
T_{n}^{\prime}(x)=-\frac{\sin (n \operatorname{arcos} x)}{2^{n-1}} \frac{1}{\sqrt{\left(1-x^{2}\right)}},
$$

and

$$
\begin{aligned}
V_{n}\left(x_{1, n}, \ldots, x_{n, n}\right) & =\left|T_{n}^{\prime}\left(x_{1, n}\right) \ldots T_{n}^{\prime}\left(x_{n, n}\right)\right| \\
& =\frac{1}{2^{n(n-1)}} \cdot \frac{1}{\sin (\pi / 2 n) \sin (3 \pi / 2 n) \ldots \sin ((2 n-1) \pi / 2 n)} .
\end{aligned}
$$


The relations

$$
\frac{2}{\pi} \theta \leqq \sin \theta \leqq 1, \quad 0 \leqq \theta \leqq \frac{\pi}{2}, \quad \sin (\pi-\theta)=\sin \theta,
$$

lead to the inequality

$$
\frac{1}{2^{n(n-1)}} \leqq V\left(x_{1, n}, \ldots, x_{n, n}\right) \leqq \frac{n^{n}}{2^{n(n-1)}},
$$

from which (3.16) follows by root extraction.

(b) We will need the estimate, valid for fixed $n$, and $i \neq j$ :

$$
\begin{aligned}
\left|x_{i, n}-x_{j, n}\right| & =\left|\cos \left(\frac{2 i-1}{2 n} \pi\right)-\cos \left(\frac{2 j-1}{2 n} \pi\right)\right| \\
& =2\left|\sin \left(\frac{i+j-1}{2 n} \pi\right) \sin \left(\frac{i-j}{2 n} \pi\right)\right| \geqq \frac{8}{n^{2}},
\end{aligned}
$$

again making use of (3.20).

It follows from (3.18) that, for $i \neq j$,

$$
A_{i, j, n}=\left|x_{i, n}-x_{j, n}\right|\left(1+\frac{\theta_{i, j, n}}{n^{3}\left|x_{i, n}-x_{j, n}\right|}\right) .
$$

Using (3.21), we then obtain

$$
\left|x_{i, n}-x_{j, n}\right|\left(1-\frac{1}{8 n}\right) \leqq A_{i, j, n} \leqq\left|x_{i, n}-x_{j, n}\right|\left(1+\frac{1}{8 n}\right) .
$$

With the use of (3.16), (3.19) is now readily obtained, completing the proof of the lemma.

We now prove our first result from $\S 1$.

Proof of Lemma 1.3. (a) The Frostman measure $\mu_{I}$ is given by (1.3), and since it is absolutely continuous with respect to linear Lebesgue measure, $I$ has stable capacity by Theorem 3.2(a). By Theorem 3.1(b), $\Delta(I)=1 / 2$.

(b) For each positive integer $n$ and real number $\delta(0<\delta \leqq 1)$, we define the set $K_{n}^{\delta}$ as follows. The set $K_{n}^{\delta}$ is the union of $n$ closed intervals $l_{i, n}^{\delta}$, each of length $\left|l_{i, n}^{\delta}\right|=\delta / n^{3}$ and each centred at a different one of the points $x_{i, n}(i=1, \ldots, n)$ introduced in Lemma 3.5. By (3.21) these intervals are pairwise disjoint and are contained in $I$. Thus $\operatorname{meas} K_{n}^{\delta}=n\left(\delta / n^{3}\right)=\delta / n^{2}$.

Let $\omega_{n}^{\delta}$ be the measure $(1 / n) \sum_{i=1}^{n} \mu_{i, n}^{\delta}$, where $\mu_{i, n}^{\delta}$ is the Frostman measure associated with $l_{i, n}^{\delta}$. By Theorem 3.1

$$
I\left(\mu_{i, n}^{\delta}\right)=\log \left(\frac{4}{\left|l_{i, n}^{\delta}\right|}\right)=\log \left(\frac{4 n^{3}}{\delta}\right) .
$$


REGULAR BEHAVIOUR OF ORTHOGONAL POLYNOMIALS 131

If $\mu$ and $\nu$ are measures defined on the measure space $(R, F)$ (Definition 3.1), let

$$
I(\mu, \nu)=\int \log \frac{1}{|z-t|} d(\mu \times \nu),
$$

where $\mu \times \nu$ is the complete product measure defined on $R \times R$. For $i \neq j$, we then obtain

where

$$
I\left(\mu_{i, n}^{\delta}, \mu_{j, n}^{\delta}\right) \leqq \log \left(1 / A_{i, j, n}^{\delta}\right),
$$

$$
A_{i, j, n}^{\delta}=\left|x_{i, n}-x_{j, n}\right|-\delta / n^{3} \text {. }
$$

Now $\omega_{n}^{\delta}$ is a unit measure with $c\left(\omega_{n}^{\delta}\right) \subset K_{n}^{\delta}$, and so

$$
\begin{aligned}
V\left(K_{n}^{\delta}\right) & \leqq I\left(\omega_{n}^{\delta}\right)=\frac{1}{n^{2}} \sum_{i=1}^{n} I\left(\mu_{i, n}^{\delta}\right)+\frac{1}{n^{2}} \sum_{\substack{i, j=1 \\
i \neq j}}^{n} I\left(\mu_{i, n}^{\delta}, \mu_{j, n}^{\delta}\right) \\
& \leqq \frac{1}{n} \log \left(\frac{4 n^{3}}{\delta}\right)+\frac{1}{n^{2}} \sum_{\substack{i, j=1 \\
i \neq j}}^{n} \log \frac{1}{A_{i, j, n}}
\end{aligned}
$$

where we have used (3.22) and (3.24).

We thus obtain

$$
C\left(K_{n}^{\delta}\right) \geqq\left(\frac{\delta}{4 n^{3}}\right)^{1 / n}\left(\prod_{\substack{i, j=1 \\ i \neq j}}^{n} A_{i, j, n}^{\delta}\right)^{1 / n^{2}} .
$$

By (3.25) and the fact that $0<\delta<1$, we can apply Lemma 3.5(b) to (3.27), and also use the fact that $C\left(K_{n}^{\delta}\right) \leqq 1 / 2$, so as to obtain $\lim _{n \rightarrow \infty} C\left(K_{n}^{\delta}\right)=1 / 2$. If $B_{\delta}=\bigcup_{n=1}^{\infty} K_{n}^{\delta}$, then meas $B_{\delta} \leqq \delta \sum_{n=1}^{\infty} 1 / n^{2}$ and $C\left(B_{\delta}\right)=1 / 2$. Thus, for $\delta_{1}=\min \left\{6 \varepsilon / \pi^{2}, 1\right\}$, where $\varepsilon>0$, meas $B_{\delta_{1}} \leqq \varepsilon$ and $C\left(B_{\delta_{1}}\right)=1 / 2$.

If we let $R_{n}^{\delta_{1}}=\bigcup_{j=1}^{n} K_{j}^{\delta_{1}}$, then $B_{\delta_{1}}=\bigcup_{n=1}^{\infty} R_{n}^{\delta_{1}}$ and $R_{n}^{\delta_{1}} \subset R_{n+1}^{\delta_{1}}(n=1, \ldots)$. By Lemma 3.4 and Theorem 3.2(a) the set $R_{n}^{\delta_{1}}$, which is a finite union of pairwise disjoint compact intervals, has stable capacity. Thus by Theorem $3.2(\mathrm{~b}), B_{\delta_{1}}$ has stable capacity. We finally observe that the points $x_{i, n}(n=1, \ldots, i=1, \ldots, n)$ are dense in $I$, from which it follows that $B_{\delta_{1}} \subset N(I)$. Thus, for a given $\varepsilon>0$, the set $B_{\delta_{1}}$ satisfies all the requirements of the set $E$ in the statement of Lemma 1.3(b), and the proof is complete.

(c) If $E_{0}=\bigcup_{n=1}^{\infty} E_{n}$, where $\left\{E_{n}\right\}(n=1, \ldots)$ are Borel sets on the interval $[-1 / 2,1 / 2]$, then ( $[9]$ p. 63),

$$
\frac{1}{V\left(E_{0}\right)} \leqq \sum_{n=1}^{\infty} \frac{1}{V\left(\bar{E}_{n}\right)} \text {. }
$$

Take for $E_{n}$ an interval of length $\left|E_{n}\right|=\left(\frac{1}{4}\right)^{k_{n}}$, where $\left\{k_{n}\right\}$ is an increasing sequence of integers to be determined. Place these intervals on 
$[-1 / 2,1 / 2]$ so that $\bigcup_{n=1}^{\infty} E_{n}$ is dense. We then have

$$
\begin{aligned}
\frac{1}{V\left(E_{0}\right)} & \leqq \sum_{n=1}^{\infty} \frac{1}{\log \left(4 /\left|E_{n}\right|\right)}=\sum_{n=1}^{\infty} \frac{1}{\log 4^{k_{n}+1}} \\
& =\frac{1}{\log 4} \sum_{n=1}^{\infty} \frac{1}{k_{n}+1},
\end{aligned}
$$

where we have used Theorem $3.1(\mathrm{~b})$ in the second term. Thus, if the series in the last term of (3.29) has sum less than 1 , we have $C\left(E_{0}\right)<1 / 4$. The set $2 E_{0}=E$, when we use the notation and results of Theorem $3.1(\mathrm{~b})$, then satisfies $E \in N(I)$ and $C(E)=2 C\left(E_{0}\right)<1 / 2$. We observe that $L(E) \leqq C(E)<1 / 2$, and so the proofs of (c) and of the lemma are complete.

\section{Measures associated with sets}

The measure $\mu_{I}$ defined in (1.3) is uniquely associated with the interval $I$ by means of conditions stated in Theorem 1.3 and also by means of conditions stated in Theorem 3.1. What we show in this section is that conditions analogous to those of Theorem 1.3 enable us to associate unique measures with Borel sets $B$ of type $F_{\sigma}, B \subset I$ (Theorem 4.2), and on using in addition the notion of lower capacity, a unique measure is associated with sets $E \in M(I)$ (Theorem 1.4). These measures play a role in the study of the zero distribution of orthogonal polynomials.

THEOREM 4.1. Given a Borel set $B$ of type $F_{\sigma}, B \subset I, C(B)=0$, there exists a measure $\mu$, with $C(\mu) \subset I$ such that the set of plus infinities of $U(\mu)$ contains $B$.

Proof of Theorem 4.1. Let $B=\bigcup_{n=1}^{\infty} K_{n}$, where $\left\{K_{n}\right\}$ consists of compact sets, $K_{n} \subset I$ and $C\left(K_{n}\right)=0(n=1, \ldots)$. Let $\mu_{n}$ be a unit measure ( [9] 76) such that $U\left(\mu_{n}\right)$ is plus infinity on $K_{n}$, and $c\left(\mu_{n}\right) \subset K_{n}(n=1, \ldots)$. The set function $\mu=\sum_{n=1}^{\infty}\left(1 / 2^{n}\right) \mu_{n}$ is again a measure ( [6] p. 230). For any $z \in \backslash I$, there is a neighbourhood of $z$, say $N(z)$, such that $N(z) \subset \backslash I$. Since $\mu_{n}(N(z))=0, \mu(N(z))=0$, and so $c(\mu) \subset I$. Let $\nu_{n}=\sum_{k=1}^{n}\left(1 / 2^{k}\right) \mu_{k}$. If $z \in I$ and $t \in I, \log (1 /|z-t|) \geqq 0$. Hence

$$
\lim _{n \rightarrow \infty} \int \log \frac{2}{|z-t|} d \nu_{n} \leqq \int \log \frac{2}{|z-t|} d \mu
$$

since the left-hand expression is non-decreasing for fixed $z$. Thus the right-hand side is plus infinity if $z \in B$, since the left-hand side is such, and the same is then true for $U(\mu)$. This completes the proof.

DEFINITION 4.1. Let $\left(\mu_{n}: \mu_{n} \in \Omega, n=1, \ldots\right)$ be a sequence of measures. We say that $\left(\mu_{n}\right)$ converges to $\mu_{0} \in \Omega$ and write 
REGULAR BEHAVIOUR OF ORTHOGONAL POLYNOMIALS 133

$\lim _{n \rightarrow \infty} \mu_{n}=\mu_{0}$ if

$$
\lim _{n \rightarrow \infty} \int f(t) d \mu_{n}=\int f(t) d \mu
$$

for all real-valued functions $f(t)$ continuous on $I$. We say, for $\mu, \nu \in \Omega$, that $\mu=\nu$ if $\mu(B)=\nu(B)$ for all Borel subsets $B$ of $I$.

LEMma 4.1 ([7] p. xiii). (a) Every infinite sequence of measures $\left(\mu_{n}\right.$ : $\left.\mu_{n} \in \Omega, n=1, \ldots\right)$, contains a convergent subsequence.

(b) If an infinite sequence of measures $\left(\mu_{n}: \mu_{n} \in \Omega, n=1, \ldots\right)$, has the property that every infinite subsequence has a further subsequence converging to $\mu_{0} \in \Omega$, then the original sequence converges to $\mu_{0}$.

LEMma 4.2 ([9] p. 34). If $\mu_{n} \in \Omega(n=0,1, \ldots)$ and $\lim _{n \rightarrow \infty} \mu_{n}=\mu_{0}$, then, for any Borel set $B \subset I$, for which the boundary of $B, \partial B$, satisfies $\mu_{0}(\partial B)=0$, we have $\lim _{n \rightarrow \infty} \mu_{n}(B)=\mu_{0}(B)$.

Proof of Lemma 1.4. Consider numbers $\alpha, \beta$, where $-1 \leqq \alpha<\beta \leqq 1$. We first note that $\nu_{n}([\alpha, \beta])=\nu_{n}^{*}(\alpha, \beta) / n$, where $\nu_{n}$ is the measure introduced in the statement of the lemma, and $\nu_{n}^{*}(\alpha, \beta)$ is defined immediately following (1.1). Now $\partial([\alpha, \beta])=\{\alpha, \beta\}$ and $\mu_{I}(\{\alpha, \beta\})=0$ according to (1.3). Thus, by Lemma 4.2 and (1.3), if (1.4) holds, then

$$
\lim _{n \rightarrow \infty} v_{n}([\alpha, \beta])=\mu_{I}([\alpha, \beta])=\frac{1}{\pi} \int_{\alpha}^{\beta} \frac{d x}{\sqrt{\left(1-x^{2}\right)}},
$$

so that (1.1) follows. This completes the proof of sufficiency.

We sketch the proof of necessity. We start with the hypothesis that, when $-1 \leqq \alpha<\beta \leqq 1, \lim _{n \rightarrow \infty} \nu_{n}([\alpha, \beta])=\mu_{I}([\alpha, \beta])$. This can be written

$$
\lim _{n \rightarrow \infty} \int f(x) d \nu_{n}=\int f(x) d \mu,
$$

where $f(x)$ is the characteristic function of $[\alpha, \beta]$. We then can extend $\left(^{*}\right)$ to the case where $f(x)$ is the characteristic function of a half-open or open interval, since $\mu_{I}$ is a continuous measure. The extension to the case where $f(x)$ is a step function follows since a step function is a finite linear combination of characteristic functions of closed, half-open, or open intervals. The final extension of $\left(^{*}\right)$ to continuous functions $f(x)$ follows since $I$ is compact, so that continuous functions can be uniformly approximated by step functions. This completes the proof of necessity.

LEMma 4.3 ([5] p. 85). If $\mu_{n} \in \Omega(n=0,1, \ldots)$ and $\lim _{n \rightarrow \infty} \mu_{n}=\mu_{0}$, then

$$
U\left(z, \mu_{0}\right) \leqq \lim _{n \rightarrow \infty} U\left(z, \mu_{n}\right)
$$


where inequality holds, if at all, only on a subset $B$ of I that is a Borel set of type $F_{\sigma}$ and of capacity zero.

This useful result is known as the lower envelope theorem for potentials.

Lemma 4.4 ([9] p. 53). (a) Let $\mu \in \Omega$. Then $U(z, \mu)$ is lower semicontinuous and superharmonic in the finite complex plane.

(b) ([9] pp. 34, 50). If $\mu_{i} \in \Omega(i=1,2)$, and $U\left(z, \mu_{1}\right)=U\left(z, \mu_{2}\right)$ for $z \in \backslash I$, then $\mu_{1}=\mu_{2}$.

Theorem 4.2. Given a Borel set $B$ of type $F_{\sigma}, B \subset I, C(B)>0$, (a) there exists a measure $\mu \in \Omega$ such that $U(\mu) \leqq \log (1 / C(B))$ for $x \in B$, where inequality holds on $B$, if at all, only for a Borel set $B_{1}$ of type $F_{\sigma}$ and of capacity zero.

(b) There is a unique measure $\mu \in \Omega$ for which $U(\mu) \geqq \log (1 / C(B))$ for $x \in B$, with the possible exception of a Borel set of type $F_{\sigma}$ and capacity 0 . In particular, therefore, there is a unique measure satisfying the requirements of (a).

Proof of Theorem 4.2. (a) If $B$ is compact, then the Frostman measure $\mu_{B}$ of Theorem 3.1(a) satisfies the requirements. Assume, therefore, that $B$ is not compact, and let $B=\bigcup_{n=1}^{\infty} K_{n}, K_{n} \subset K_{n+1}$, where $\left\{K_{n}\right\}(n=1, \ldots)$ consists of compact sets. We can assume that $C\left(K_{n}\right)>0$ without loss of generality (see proof of Lemma 3.2). Also, by Lemma 3.2,

$$
\lim _{n \rightarrow \infty} C\left(K_{n}\right)=C(B) .
$$

Let $\mu_{n}=\mu_{K_{n}}$ be the Frostman measure associated with $K_{n}$. Let $\left\{k_{n}\right\}$ be an increasing sequence of integers for which $\left\{\mu_{k_{n}}\right\}$ converges, say to the limit $\mu_{0}$. The existence of such a sequence is a consequence of Lemma 4.1. By Lemma 4.3

$$
U\left(x, \mu_{0}\right) \leqq \varliminf_{n \rightarrow \infty} U\left(x, \mu_{k_{n}}\right),
$$

where equality holds except for a Borel set $B_{2}, B_{2} \subset I$, of type $F_{\sigma}$ and capacity zero. We next establish that the right side of (4.1) has the value $\log (1 / C(B))$ for $x \in B$, except for a set $B_{3}$ that is a Borel set of type $F_{\sigma}$ and capacity zero. Thus on $B$, except for the set $B_{1}=B \cap\left(B_{2} \cup B_{3}\right)$, again a Borel set of type $F_{\sigma}$ and capacity zero,

$$
U\left(x, \mu_{0}\right)=\log \frac{1}{C(B)} .
$$

We have used in this argument Lemma 3.1(a) to show that $B_{2} \cup B_{3}$ has capacity zero, and now must establish the existence of the set $B_{3}$ with the desired properties. 
By Theorem 3.1(a) $U\left(x, \mu_{k_{n}}\right)=\log \left(1 / C\left(K_{k_{n}}\right)\right)$ for $x \in K_{k_{n}}$, except for the set $B^{k_{n}}$, a Borel set of type $F_{\sigma}$ and capacity zero. Thus, if $B_{3}=\bigcup_{n=1}^{\infty} B^{k_{n}}$, then $B_{3}$ is again an $F_{\sigma}$ set and, by Lemma 3.1 , has capacity zero. Since $\lim _{n \rightarrow \infty} C\left(K_{k_{n}}\right)=C(B), \lim _{n \rightarrow \infty} U\left(x, \mu_{k_{n}}\right)=\log (1 / C(B))$ if $x \in B \backslash B_{3}$, and so the proof of (a) is complete.

(b) Let $\nu \in \Omega$ be a measure for which $U(x, \nu) \geqq \log (1 / C(B))$ for $x \in B$, except for a Borel set $B_{4}$ of type $F_{\sigma}$ and capacity zero. Our object is to prove that $\nu=\mu_{0}$, the measure introduced in (a), thus proving uniqueness.

Let $\mu^{*}$ be a measure, which exists by Theorem 4.1, such that $U\left(x, \mu^{*}\right)=\infty$ for $x \in B_{4}$. Setting aside the case where $B$ is compact for the time being, we can represent $B$ as $\bigcup_{n=1}^{\infty} K_{k_{n}}$, where $\left\{k_{n}\right\}$ is the sequence introduced in (a) for which $\lim \mu_{k_{n}}=\mu_{0}$. For any $\varepsilon>0$ and fixed $n>0$, form the function

$$
v_{n}^{\varepsilon}(z)=U(z, \nu)-U\left(z, \mu_{k_{n}}\right)+\varepsilon\left(U\left(z, \mu^{*}\right)-U\left(z, \mu_{k_{n}}\right)\right)
$$

This function is superharmonic in $\Delta_{n}=\left(\backslash K_{k_{n}}\right) \cup\{\infty\}$, if we define $v_{n}^{\varepsilon}(\infty)=0$. This is because the first and third terms on the right are superharmonic in the finite plane by Lemma 4.4, the second and fourth terms is harmonic in $\backslash K_{k_{n}}$ and the combination of the four terms on the right is harmonic in a deleted neighbourhood of infinity and has the limit zero as $z$ tends to infinity. Thus $\infty$ can be removed as a singularity by letting $v_{n}^{8}(\infty)=0$. It then follows ([3] 75) that, if, for all $\zeta \in K_{k_{n}}$ and for all sequences $\left(z_{k}: z_{k} \in \Delta_{n}\right), \lim _{k \rightarrow \infty} z_{k}=\zeta$, we have

$$
\varliminf_{k \rightarrow \infty} v_{n}^{\varepsilon}\left(z_{k}\right) \geqq A_{n}^{e},
$$

then, for $z \in \Delta_{n}$,

$$
v_{n}^{\varepsilon}(z) \geqq A_{n}^{\varepsilon}
$$

Our task now is to find a suitable value for $A_{n}^{\varepsilon}$.

To begin with, by Theorem $3.1(\mathrm{a}), U\left(z, \mu_{k_{n}}\right) \leqq \log \left(1 / C\left(K_{k_{n}}\right)\right)$ for all finite $z$, so that

$$
v_{n}^{\varepsilon}(z) \geqq U(z, \nu)-\log \left(1 / C\left(K_{k_{n}}\right)\right)+\varepsilon\left(U\left(z, \mu^{*}\right)-\log \left(1 / C\left(K_{k_{n}}\right)\right)\right) .
$$

If $\left(z_{k}\right)$ is any sequence with limit $\zeta \in K_{k_{n}}$, then

$$
\begin{aligned}
\lim _{k \rightarrow \infty} v_{n}^{\varepsilon}\left(z_{k}\right) & \geqq \lim _{k \rightarrow \infty} U\left(z_{k}, \nu\right)-\log \left(1 / C\left(K_{k_{n}}\right)\right)+\varepsilon\left(\lim _{k \rightarrow \infty} U\left(z_{k}, \mu^{*}\right)-\log \left(1 / C\left(K_{k_{n}}\right)\right)\right) \\
& \geqq U(\zeta, \nu)-\log \left(1 / C\left(K_{k_{n}}\right)\right)+\varepsilon\left(U\left(\zeta, \mu^{*}\right)-\log \left(1 / C\left(K_{k_{n}}\right)\right)\right),
\end{aligned}
$$

where the lower semi-continuity of potentials (Lemma 4.4) is used to obtain the second inequality. For any $\mu \in \Omega$ and $x \in I$, we have

$$
U(x, \mu)=\int \log \frac{1}{|x-t|} d \mu \geqq \log \frac{1}{2} .
$$


Thus, for $\zeta \in B_{4}$, the last collection of terms in (4.4) is plus infinity and a lower bound of this collection of terms for $\zeta \in K_{k_{n}} \backslash B_{4}$ can be chosen as $A_{n}^{\varepsilon}$, namely

$$
A_{n}^{s}=\log (1 / C(B))-\log \left(1 / C\left(K_{k_{n}}\right)\right)+\varepsilon\left(\log \frac{1}{2}-\log \left(1 / C\left(K_{k_{n}}\right)\right)\right) .
$$

Thus, from (4.3), we obtain

$$
U(z, \nu)-U\left(z, \mu_{k_{n}}\right) \geqq-\varepsilon\left(U\left(z, \mu^{*}\right)-U\left(z, \mu_{k_{n}}\right)\right)+A_{n}^{\varepsilon}
$$

For $z \in \backslash I, U\left(z, \mu^{*}\right)$ and $U\left(z, \mu_{k_{n}}\right)$ are finite, so we must have, on letting $\varepsilon \rightarrow 0$,

$$
U(z, v)-U\left(z, \mu_{k_{n}}\right) \geqq \log (1 / C(B))-\log \left(1 / C\left(K_{k_{n}}\right)\right) .
$$

Since $\lim _{n \rightarrow \infty} \mu_{k_{m}}=\mu_{0}$ and $\log (1 /|z-t|)$ is continuous in $t$ for $t \in I$ and $z \in \backslash I$, by Lemma 4.2 we obtain, on letting $n$ tend to infinity in (4.5),

$$
U(z, \nu)-U\left(z, \mu_{0}\right) \geqq 0 .
$$

The left side is harmonic in $\backslash I$ and can be made harmonic in $\backslash I \cup\{\infty\}$ by assigning the value zero at infinity, which is the limiting value. But for this extended function, the value at infinity is equal to the lower bound of values on $\backslash I$, so that

$$
U(z, v)-U\left(z, \mu_{0}\right)=0 \quad(z \in \backslash I),
$$

and, by Lemma 4.4, $\nu=\mu_{0}$. Thus we have reached our objective for the case where $B$ is not compact.

When $B$ is compact, we replace $\mu_{k_{n}}$ in (4.2) by $\mu_{B}$. The argument proceeds as before, $\mu_{B}$ replacing $\mu_{0}$. This completes the proof of the theorem.

Proof of Lemma 1.5. Using the notation introduced in Lemma 1.4, we take the negative of the logarithm on both sides of (1.8) and must prove that

$$
\lim _{n \rightarrow \infty} U\left(z, \nu_{n}\right)=\log \frac{1}{|f(z)|} \quad\left(z \in C_{1}\right)
$$

implies (1.4). We next identify $\log (1 /|f(z)|)$ with $U\left(z, \mu_{I}\right)$ for $z \in C_{1}$ as follows. Their difference $D(z)$ is harmonic in $C_{1}$ and, if extended to infinity by assigning the limit, which is zero, the difference is harmonic in $\Delta=C_{1} \cup\{\infty\}$. For $\zeta \in I, z_{n} \in \Delta(n=1, \ldots), \lim _{n \rightarrow \infty} z_{n}=\zeta$, we have $\lim _{n \rightarrow \infty}\left|f\left(z_{n}\right)\right|=1 / 2$ and $\lim _{n \rightarrow \infty} U\left(z_{n}, \mu_{I}\right)=\log 2:$ thus $\lim _{n \rightarrow \infty} D\left(z_{n}\right)=0$. The harmonic property of $D(z)$ in $\Delta$ then gives $D(z) \geqq 0$ for $z \in C_{1}$; but since $D(\infty)=0$, it follows that $D(z)=0$ for $z \in C_{1}$. Thus (4.7) becomes

$$
\lim _{n \rightarrow \infty} U\left(z, v_{n}\right)=U\left(z, \mu_{I}\right) \quad\left(z \in C_{1}\right) .
$$


By Lemma 4.1 (a) every infinite subsequence of $\left(v_{n}\right)$ has a convergent subsequence, say $\left(\nu_{k_{n}}\right)$ with limit $\mu_{0}$. If we can show that $\mu_{0}=\mu_{I}$, then, by Lemma 4.1 (b), (1.4) follows. If $z \in C_{1}, \log (1 /|z-t|)$ is a continuous function of $t$ when $t \in I$. Hence, by Definition 4.1 and (4.8),

$$
\lim _{n \rightarrow \infty} U\left(z, v_{k_{n}}\right)=U\left(z, \mu_{0}\right)=U\left(z, \mu_{I}\right)
$$

if $z \in C_{1}$. Thus, by Lemma $4.4(\mathrm{~b}), \mu_{0}=\mu_{I}$, thus completing the proof of sufficiency.

We sketch the proof of necessity. If the polynomials in the set $\left\{P_{n}(x)\right\}$ have regular behaviour, then, by Lemma 1.3 , the measures $\nu_{n}$ converge to $\mu_{I}$. Hence relationship (4.8) holds and this implies (4.7), which is equivalent to (1.8), completing the proof of necessity.

Proof of Theorem 1.3. By Theorem 4.2 (b) there is a unique measure $\nu$ such that $U(x, \nu) \geqq \log 2$ for $x \in I$, with the possible exception of a Borel set of capacity zero. By Theorem 3.1 (b), $U\left(x, \mu_{I}\right)=\log 2$ for $x \in I$. Thus $\nu=\mu_{I}$, which completes the proof.

Proof of Theorem 1.4. We first note that by Lemma $3.3(\mathrm{~b}), L(E)>0$. Suppose that $\mu$ is a measure such that $U(x, \mu) \geqq \log (1 / L(E))$ for $x \in E_{1}$, an equimeasurable subset of $E$. By Lemma 3.3 (a) there is an equimeasurable subset of $E$, say $E_{2}$, with $C\left(E_{2}\right)=L(E)$. We now note that $E_{3}=E_{1} \cap E_{2}$ is an equimeasurable subset of $E$ of stable capacity $L(E)$ on which $U(x, \mu) \geqq \log (1 / L(E))$. Let $B_{1}$ be an equimeasurable subset of $E_{3}$ which is a Borel set of type $F_{\sigma}$. Then $C\left(B_{1}\right)=L(E)$ and hence $U(z, \mu) \geqq \log \left(1 / C\left(B_{1}\right)\right)$ for $x \in B_{1}$. Thus $\mu$ is the measure uniquely associated with $B_{1}$ by Theorem 4.2.

Let $\nu$ be any measure such that $U(x, \nu) \geqq \log (1 / L(E))$ on $E_{4}$, an equimeasurable subset of $E$. Our object is to show that $\nu=\mu$, thus proving uniqueness. The set $E_{5}=E_{4} \cap E_{2}$ has stable capacity $L(E)$ and contains an equimeasurable subset $B_{2}$ which is a Borel set of type $F_{\sigma}$ with $C\left(B_{2}\right)=L(E)$. Thus $U(x, \nu) \geqq \log \left(1 / C\left(B_{2}\right)\right)$ for $x \in B_{2}$ and so $\nu$ is the unique measure associated with $B_{2}$ by Theorem 4.2. Now the set $B_{3}=B_{1} \cap B_{2}$ is a Borel set of type $F_{\sigma}$ and an equimeasurable subset of $E_{2}$ with $C\left(B_{3}\right)=L(E)$. Also both $U(x, \nu)$ and $U(x, \mu)$ are not less than $\log \left(1 / C\left(B_{3}\right)\right)$ for $x \in B_{3}$. Hence, by Theorem $4.2, \nu=\mu$, since they are both the unique measure associated with $B_{3}$ by Theorem 4.2. This proves uniqueness. When the measure exists, we denote it by $\mu_{E}^{*}$.

If the measure $\mu_{E}^{*}$ exists, it is the unique measure associated with $B_{1}$. Then, by Theorem $4.2(\mathrm{a}), U\left(x, \mu_{E}^{*}\right)=\log (1 / L(E))$ for $x \in B_{1}$, except possibly for a Borel set $B_{4}$ of capacity zero. Thus equality holds on 
$B=B_{1} \backslash B_{4}$, which is a Borel set which satisfies $C(B)=L(E)$ by Lemma $3.1(\mathrm{~b})$.

If $L(E)<1 / 2$, we have just seen that $U\left(x, \mu_{E}^{*}\right)=\log (1 / L(E))>\log 2$ on a Borel set $B, B \subset I$, with $C(B)=L(E) . U\left(x, \mu_{I}\right)$, on the other hand, by Theorem 3.1 (a) equals $\log 2$ for $x \in I$. This could not happen if $\mu_{I}=\mu_{E}^{*}$, so $\mu_{I} \neq \mu_{E}^{*}$. If $L(E)=1 / 2$, then $U\left(x, \mu_{I}\right)=\log 2=\log (1 / L(E))$ for $x \in I$, hence for $x \in E$. By what has been shown, there is a unique measure with the property, so $\mu_{E}^{*}=\mu_{I}$. This completes the proof of the theorem.

\section{Norm behaviour and zero distribution}

Lemma 5.1 ([9] p. 72). Let $K \subset I$ be a compact set. For each integer $n, n \geqq 0$, there is a unique monic polynomial of degree $n, T_{n}(x \mid K)$, such that

$$
\sup _{x \in \boldsymbol{K}}\left|T_{n}(x \mid K)\right|<\sup _{x \in \mathcal{K}}\left|Q_{n}(x)\right|,
$$

where $Q_{n}(x)$ is any other monic polynomial of degree $n$. If

$$
M_{n}(K)=\sup _{x \in K}\left|T_{n}(x \mid K)\right|,
$$

then

$$
\lim _{n \rightarrow \infty}\left|M_{n}(K)\right|^{1 / n}=C(K) .
$$

All the zeros of $T_{n}(x \mid K)$ lie in $I$. The polynomial $T_{n}(x \mid I)$ is the same as the polynomial $T_{n}(x)$ introduced in Lemma 3.5 .

Lemma 5.2 ([8] p. 38). For $p(x) \in P$ and any integer $n>0$,

$$
\int\left|P_{n}(x \mid p)\right|^{2} p(x) d x<\int\left|Q_{n}(x)\right|^{2} p(x) d x,
$$

where $Q_{n}(x)$ is any monic polynomial of degree $n$ other than $P_{n}(x \mid p)$, the orthogonal polynomial of degree $n$ associated with the weight function $p(x)$.

For $\mu \in \Omega$ with $c(\mu)$ an infinite set (Theorem 1.8), we have

$$
\int\left|P_{n}(x \mid \mu)\right|^{2} d \mu<\int\left|Q_{n}(x)\right|^{2} d \mu,
$$

where $Q_{n}(x)$ is any monic polynomial of degree $n$ other than $P_{n}(x \mid \mu)$, the orthogonal polynomial of degree $n$ associated with $\mu$.

Proof of Lemma 1.2. If $p(x) \in P, p \notin N(I)$, there is a pair of real numbers $\alpha, \beta$, such that $-1 \leqq \alpha<\beta \leqq 1$ and $\int_{\alpha}^{\beta} p(x) d x=0$. We will show that then $P_{n}(x \mid p)$ can have at most one zero in $(\alpha, \beta)(n=1, \ldots)$. On the other hand, it follows from (1.1) that, if $p(x)$ is regular, then $\nu_{n}^{*}(\alpha, \beta)$ tends to infinity. Thus $p(x)$ cannot be regular. 
REGULAR BEHAVIOUR OF ORTHOGONAL POLYNOMIALS 139

If, for any $n>0, P_{n}(x \mid p)$ has two zeros in $(\alpha, \beta)$, say $x_{1}, x_{2}$, where $-1 \leqq \alpha<x_{1}<x_{2}<\beta \leqq 1$, we consider the modified polynomial

$$
P_{n}^{*}(x)=P_{n}(x \mid p) \frac{\left(x-\left(x_{1}-\varepsilon\right)\right)\left(x-\left(x_{2}+\varepsilon\right)\right)}{\left(x-x_{1}\right)\left(x-x_{2}\right)},
$$

where $\varepsilon>0$ is chosen so that $\alpha<x_{1}-\varepsilon<x_{2}+\varepsilon<\beta$.

Now

$$
\begin{aligned}
\frac{\left(x-x_{1}+\varepsilon\right)\left(x-x_{2}-\varepsilon\right)}{\left(x-x_{1}\right)\left(x-x_{2}\right)} & =\left(1+\frac{\varepsilon}{x-x_{1}}\right)\left(1-\frac{\varepsilon}{x-x_{2}}\right) \\
& =1+\varepsilon\left(\frac{x_{1}-x_{2}}{\left(x-x_{1}\right)\left(x-x_{2}\right)}\right)-\frac{\varepsilon^{2}}{\left(x-x_{1}\right)\left(x-x_{2}\right)}
\end{aligned}
$$

and, for $\left(x-x_{1}\right)\left(x-x_{2}\right)>0$, the last group of terms in (5.3) is less than 1 . Thus $\left|P_{n}^{*}(x)\right|<\left|P_{n}(x)\right|$ in $I \backslash[\alpha, \beta]$, and so

$$
\begin{aligned}
\int_{I}\left|P_{n}^{*}(x)\right|^{2} p(x) d x & =\int_{I \backslash[\alpha, \beta]}\left|P_{n}^{*}(x)\right|^{2} p(x) d x \\
& \leqq \int_{I \backslash \alpha, \beta]}\left|P_{n}(x \mid p)\right|^{2} p(x) d x \\
& =\int_{I}\left|P_{n}(x \mid p)\right|^{2} p(x) d x
\end{aligned}
$$

which contradicts (5.1).

LEMMA 5.3. Let $\left(f_{n}(x): n=1, \ldots\right)$, be a sequence of non-negative, Borel measurable functions defined on $I$, and let $\left(k_{n}\right)$ be an increasing sequence of integers. If

$$
\varlimsup_{n \rightarrow \infty}\left(\int_{I} f_{k_{n}}(x) d x\right)^{1 / k_{n}} \leqq 1,
$$

then there is an infinite subsequence $\left(t_{n}\right)$ of $\left(k_{n}\right)$, and a Borel set $B$ of measure zero such that

$$
\varlimsup_{n \rightarrow \infty}\left(f_{t_{n}}(x)\right)^{1 / n_{n}} \leqq 1
$$

for $x \in I \backslash B$.

Proof of Lemma 5.3. There is a sequence $\left(\varepsilon_{k_{n}}\right)$, with $\varepsilon_{k_{n}}>0(n=1, \ldots)$ and $\lim _{n \rightarrow \infty} \varepsilon_{k_{n}}=0$, such that

$$
\left(\int_{I} f_{k_{n}}(x) d x\right)^{1 / k_{n}} \leqq 1+\varepsilon_{k_{n}} \quad(n=1, \ldots) .
$$

Thus

$$
\int_{I} f_{k_{n}}(x) d x \leqq\left(1+\varepsilon_{k_{n}}\right)^{k_{n}}
$$


and

$$
\int_{I} g_{k_{n}}(x) d x \leqq 1 / k_{n}
$$

where

$$
g_{k_{n}}(x)=\frac{f_{k_{n}}(x)}{k_{n}\left(1+\varepsilon_{k_{n}}\right)^{k_{n}}} .
$$

It follows from (5.5) ([6] p. 91) that there is a subsequence of $\left(k_{n}\right)$, say $\left(t_{n}\right)$, and a set $B$ of measure zero, such that

$$
\lim _{n \rightarrow \infty} g_{l_{n}}(x)=0
$$

for $x \in I \backslash B$. Since $g_{l_{n}}(x)$ is Borel measurable, the function $\varlimsup_{n \rightarrow \infty} g_{h_{n}}(x)$ is Borel measurable, and thus

$$
B=\left\{x: \varlimsup_{n \rightarrow \infty} g_{l_{n}}(x)>0\right\}
$$

is a Borel set. Also, for $x \in I \backslash B$, there is an integer $n(x)$ such that

$$
g_{h_{n}}(x)=\frac{f_{l_{n}}(x)}{t_{n}\left(1+\varepsilon_{l_{n}}\right)^{l_{n}}}<1
$$

for $n>n(x)$. Thus, for $n>n(x)$,

$$
f_{l_{n}}(x) \leqq t_{n}\left(1+\varepsilon_{l_{n}}\right)^{l_{n}}
$$

so that we obtain (5.4) by root extraction. This completes the proof of the lemma.

LEMma 5.4. Let $K_{i} \subset I$ be compact sets $(i=0,1)$, with $K_{0} \cap K_{1}=\emptyset$, and $C\left(K_{i}\right)>0(i=0,1)$. Then

$$
C\left(K_{0}\right)<C\left(K_{0} \cup K_{1}\right)
$$

Proof of Lemma 5.4. Let $\mu_{i}$ represent the Frostman measure of $K_{i}$ $(i=0,1)$. We will show that

$$
V\left(K_{0} \cup K_{1}\right)<V\left(K_{0}\right)
$$

from which (5.7) follows. If $0 \leqq \varepsilon \leqq 1$, let $\mu_{\varepsilon}=(1-\varepsilon) \mu_{0}+\varepsilon \mu_{1}$. Since $c\left(\mu_{\varepsilon}\right) \subset K_{0} \cup K_{1}$, we have

$$
\begin{aligned}
V\left(K_{0} \cup K_{1}\right) & \leqq I\left(\mu_{\varepsilon}\right) \\
& =(1-\varepsilon)^{2} I\left(\mu_{0}\right)+\varepsilon^{2} I\left(\mu_{1}\right)+2(\varepsilon)(1-\varepsilon) I\left(\mu_{0}, \mu_{1}\right),
\end{aligned}
$$

the last term being defined in (3.23). By Fubini's theorem, we can write

$$
I\left(\mu_{0}, \mu_{1}\right)=\int U\left(\mu_{0}\right) d \mu_{1} .
$$


REGULAR BEHAVIOUR OF ORTHOGONAL POLYNOMIALS 141

Now $U\left(\mu_{0}\right)$ is continuous on $K_{1}$, and $\max U\left(\mu_{0}\right)=\alpha<V\left(K_{0}\right)$ by Theorem 3.1 (a). Thus, from (5.9), we have $x \in \mathcal{K}_{1}$

$$
\begin{aligned}
V\left(K_{0} \cup K_{1}\right) & \leqq(1-\varepsilon)^{2} V\left(K_{0}\right)+\varepsilon^{2} V\left(K_{1}\right)+2 \varepsilon(1-\varepsilon) \alpha \\
& =V\left(K_{0}\right)-2 \varepsilon\left(V\left(K_{0}\right)-\alpha\right)+\varepsilon^{2}\left(V\left(K_{0}\right)+V\left(K_{1}\right)-2 \alpha\right) .
\end{aligned}
$$

Thus for sufficiently small $\varepsilon$ we obtain (5.8).

Proof of Theorem 1.5. To prove the third inequality of (1.5) consider

$$
\begin{aligned}
\left(N_{n}(p)\right)^{2} & =\int_{I}\left|P_{n}(x)\right|^{2} p(x) d x \leqq \int_{I}\left|T_{n}(x \mid I)\right|^{2} p(x) d x \\
& \leqq\left(1 / 2^{n-1}\right)^{2} \int_{I} p(x) d x,
\end{aligned}
$$

in which we have used Lemma 5.2 and Lemma 3.5 (a). We then obtain the result by root extraction.

We next suppose that the first inequality of (1.5) is violated, and deduce a contradiction. By our assumption, there is an increasing sequence of integers, $\left(k_{n}\right)$, and there is a real $\alpha$, such that

$$
\lim _{n \rightarrow \infty}\left(N_{k_{n}}(p)\right)^{1 / k_{n}} \leqq \alpha<L(E) .
$$

Since $L(E)>0$ by Lemma 3.3 , we can assume that $\alpha>0$. We can also assume that $p(x)$ is a Borel measurable function without loss of generality. Thus

$$
\lim _{n \rightarrow \infty}\left(\int_{1} \frac{\left|P_{k n}(x \mid p)\right|^{2} p(x)}{\alpha^{2 k_{n}}}\right)^{1 /\left(2 k_{n}\right)} \leqq 1 .
$$

Thus, by Lemma 5.3, there is a subsequence of $\left(2 k_{n}\right)$, say $\left(2 t_{n}\right)$, and a Borel set $B$ of measure zero, such that for $x \in I \backslash B$

$$
\varlimsup_{n \rightarrow \infty}\left(\frac{\left|P_{l n}(x \mid p)\right|^{2} p(x)}{\alpha^{2 l_{n}}}\right)^{1 /\left(2 l_{n}\right)} \leqq 1 .
$$

Since $\mathrm{S}(p(x))=E$, for $x \in E \backslash B$ it then follows that

$$
\varlimsup_{n \rightarrow \infty} \mid P_{l_{n}}(x \mid p)^{1 / l_{n}} \leqq \alpha .
$$

We next let $\nu_{l_{n}}$ be the measures associated with $P_{t_{n}}(x \mid p)$ by Lemma 1.4 . Let $\left(s_{n}\right)$ be a subsequence of $\left(t_{n}\right)$, which exists by Lemma 4.1 (a), for which $\left(\nu_{s_{n}}\right)$ converges, say with limit $\mu_{0}$. By Lemma 4.3 we have

$$
U\left(\mu_{0}\right) \leqq \lim _{n \rightarrow \infty} U\left(z, v_{s_{n}}\right)=\lim _{n \rightarrow \infty} \log \frac{1}{\left|P_{s_{n}}(z \mid p)\right|^{1 / s_{n}}},
$$

where equality holds in the first inequality except for $B_{1} \subset I$, a Borel set of capacity zero. Thus on $E \backslash\left(B \cup B_{1}\right)$, we have

$$
U\left(\mu_{0}\right) \geqq \log (1 / \alpha)>\log (1 / L(E)) .
$$


Since $B_{1}$ has capacity zero, it has measure zero ( $[9]$ p. 59), and hence $B \cup B_{1}$ has measure zero. If we let $E^{\prime}=E \backslash\left(B \cup B_{1}\right)$, then $L\left(E^{\prime}\right)=L(E)$ and $E^{\prime} \subset N(I)$. Thus $U\left(\mu_{0}\right)>\log \left(1 / L\left(E^{\prime}\right)\right)$ for $x \in E^{\prime}$. By Theorem 1.4, $U\left(\mu_{0}\right)=\log \left(1 / L\left(E^{\prime}\right)\right)$ would have to hold on a subset of $E^{\prime}$, say $B_{2}$, that is a Borel set and of the same measure as $E^{\prime}$. Since $E^{\prime}$ has positive measure, $B_{2}$ cannot be the null set, and so we have a contradiction, and the proof is complete.

Proof of Theorem $1.6(\mathrm{~b})$. We start with the sequence $\left(k_{n}\right)$ for which (1.6) holds. What we will show is that, for any subsequence $\left(t_{n}\right)$, there is a further subsequence $\left(s_{n}\right)$ for which $\lim _{n \rightarrow \infty} v_{s_{n}}=\mu_{E}^{*}$. Hence by Lemma 4.1 (b), (1.7) follows.

Once $\left(t_{n}\right)$ is chosen, choose a subsequence $\left(r_{n}\right)$ such that

$$
\varlimsup_{n \rightarrow \infty}\left|P_{r_{n}}(x, p)\right|^{1 / r_{n}} \leqq L(E)
$$

for $x \in \mathrm{S}(p(x)) \backslash B$, where $B$ is a Borel set of capacity zero. This can be achieved by applying the argument of the proof of Theorem 1.5. In (5.10) we can put $\alpha=L(E)$, and then repeat the steps indicated to (5.11), which yields (5.13). Next, choose $\left(s_{n}\right)$ as a subsequence of $\left(t_{n}\right)$ for which $\left(v_{s_{n}}\right)$ converges, say to $\mu_{0}$. Continuing the steps of the proof of Theorem 1.5 to (5.12), we now have

$$
U\left(\mu_{0}\right) \geqq \log (1 / L(E))
$$

for $x \in \mathrm{S}(p(x)) \backslash\left(B_{2} \cup B_{1}\right)$. In this case the measure $\mu_{0}$ does exist, and by Theorem 1.4 it is unique. Thus $\mu_{0}=\mu_{E}^{*}$ and as already indicated, the proof of (b) is complete.

(a) We know that $L(E)>0$ by Lemma 3.3 (b), and for convenience we let $L(E)=\alpha$. If $\alpha=1 / 2$, then, by Theorem 1.5, for any $p(x) \in P(E)$ the choice $k_{n}=n(n=1, \ldots)$ satisfies (1.6). Hence we assume that $\alpha<1 / 2$.

There is an equimeasurable subset of $E$, say $E_{1}$, of stable capacity $\alpha$ by Lemma 3.3(a). Then $E_{1}$ has an equimeasurable subset $B$ such that $B \in N(I) ; B$ is a Borel set of type $F_{\sigma}$ and $L(B)=C(B)=\alpha$. We will define a function $p(x)$ with $\mathrm{S}(p(x))=B$, and construct an increasing sequence $\left(k_{n}\right)$ such that (1.6) is satisfied. Since $p(x)$ can be extended to $E$ without affecting the value of $N_{n}(p)$, (a) will be proved.

We next show that in a representation $B=\bigcup_{n=1}^{\infty} K_{n}$, with $K_{n} \subset I$ $(n=1, \ldots), K_{n}$ a compact set, $C\left(K_{n}\right)>0, K_{n} \subset K_{n+1}$, which will exist, we must have, for any $n, C\left(K_{n}\right)<C(B)$. The set $I K_{n}$ must contain an interior point of $I$, since otherwise $C(B)=1 / 2$, and hence there is an interval $[\alpha, \beta]$ in $I$ at a positive distance from $K_{n}$. Now $B \in N(I)$, so that meas $(B \cap[\alpha, \beta])>0$, and thus $[\alpha, \beta]$ contains a compact set $K^{*}, K^{*} \subset B$, 
REGULAR BEHAVIOUR OF ORTHOGONAL POLYNOMIALS 143 meas $K^{*}>0$. By ([9] p. 59) it then follows that $C\left(K^{*}\right)>0$. By the monotonicity of capacity, $C(B) \geqq C\left(K_{n} \cup K^{*}\right)$ and, by Lemma 5.4, $C\left(K_{n} \cup K^{*}\right)>C\left(K_{n}\right)$.

Choose a representation $B=\bigcup_{n=1}^{\infty} K_{n}$ introduced in the previous paragraph. By Lemma 5.1 and the result $C\left(K_{n}\right)<C(B)$, for each integer $n>0$ there is an integer $k_{n}$ and an $\varepsilon_{n}>0$ such that

$$
\left(M_{p}\left(K_{n}\right)\right)^{1 / p} \leqq C\left(K_{n}\right)+\varepsilon_{n} \leqq C(B) \quad\left(p \geqq k_{n}\right) .
$$

There is no loss in generality in assuming that $\left(k_{n}: n=1, \ldots\right)$ is an increasing sequence of positive integers. Define a function $w(x)$ on $B$ as follows. For $x \in K_{n+1} \backslash K_{n}(n=0,1, \ldots)$, with $K_{0}=\varnothing$, let $w(x)=\left(\frac{1}{2} C(B)\right)^{k_{n}}$. Thus $w(x)$ is a Borel measurable function defined on $B$ satisfying $0<w(x)<1$. By Lemma 5.1, $\left|T_{k_{n}}\left(x \mid K_{n}\right)\right| \leqq 2^{k_{n}}$ for $x \in I$. We use only the fact that the zeros are on $I$. Also, from the definition of $w(x)$,

$$
\sup _{x \in B \backslash K_{n}} w(x)=\sup _{x \in K_{n+1} \backslash K_{n}} w(x),
$$

since the sequence $\left\{\left(\frac{1}{2} C(B)\right)^{k_{n}}\right\}$ is decreasing. Thus we obtain inequalities, for each $n>0$, where we put

$$
\begin{aligned}
g_{n}(x) & =\left|w(x) T_{k_{n}}\left(x \mid K_{n}\right)\right|, \\
\sup _{x \in B} g_{n}(x) & \leqq \max \left\{\sup _{x \in K_{n}} g_{n}(x), \sup _{x \in B \backslash K_{n}} g_{n}(x)\right\} \\
& \leqq \max \left\{(C(B))^{k_{n}},\left(\frac{1}{2} C(B)\right)^{\left.k_{n} 2^{k_{n}}\right\}}\right. \\
& =(C(B))^{k_{n}} .
\end{aligned}
$$

We next let $p(x)=(w(x))^{2}$ and note that $\mathrm{S}(p(x))=B$. We have, for $n>0$,

$$
N_{k_{n}}^{2}(p) \leqq \int_{I}\left|T_{k_{n}}\left(x \mid K_{n}\right)\right|^{2}(w(x))^{2} d x \leqq 2(C(B))^{2 k_{n}},
$$

where the first inequality follows from Lemma 5.2 and the second from (5.6). From (5.7) we have

$$
\varlimsup_{n \rightarrow \infty}\left(N_{k_{n}}(p)\right)^{1 / k_{n}} \leqq C(B)=L(E) .
$$

Since

$$
\varliminf_{n \rightarrow \infty}\left(N_{k_{n}}(p)\right)^{1 / k_{n}} \geqq L(E),
$$

by (1.5), we finally arrive at (1.6).

This completes the proof of (a) and of the Theorem.

Proof of Theorem 1.1. Since $I$ has stable capacity by Lemma 1.3, and $m(E)=2, E$ also has stable capacity $1 / 2$, so that $L(E)=1 / 2$. By Theorem 1.5, for any $p(x) \in P(E), \quad \lim _{n \rightarrow \infty}\left(N_{n}(p)\right)^{1 / n}=1 / 2 . \quad$ Thus by 
Theorem 1.6 (b) and the last part of Theorem 1.4, $\lim _{n \rightarrow \infty} v_{n}=\mu_{I}$, and thus $p(x)$ is regular by Lemma 1.4. This concludes the proof.

Proof of Theorem 1.2. The proof of sufficiency is modelled exactly on the proof of Theorem 1.1, if we pick up the argument of the proof from the point where it is shown that $L(E)=1 / 2$. For the proof of necessity, suppose that $E \in N(I)$ and $L(E)<1 / 2$. By Theorem 1.6 (a), there are $p(x) \in P(E)$ and an increasing sequence of integers $\left(k_{n}\right)$ such that $\lim _{n \rightarrow \infty}\left(N_{k_{n}}(p)\right)^{1 / k_{n}}=L(E)$. Thus, by Theorem 1.6 (b), $\lim _{n \rightarrow \infty} \nu_{k_{n}}=\mu_{E}^{*}$. By Theorem 1.4, $\mu_{E}^{*} \neq \mu_{I}$, since $L(E) \neq 1 / 2$. Hence the $p(x)$ constructed is not regular. This concludes the proof.

\section{A sufficient condition for regularity with applications}

Lemma 6.1. Let $\left\{Q_{n}(x)\right\}(n=1, \ldots)$ be a sequence of polynomials, $Q_{n}(x)=x^{n}+\ldots$, with zeros $\left\{x_{1, n}, \ldots, x_{n, n}\right\}$ on $I$. We follow the convention that a zero of multiplicity $k$ is repeated $k$ times. Let $v_{n}$ be the measure defined on the Borel subsets of $I$ determined by the conditions $\nu_{n}(\{x\})=k / n$, when $x$ is a zero of $Q_{n}(x)$ of multiplicity $k$ and $\nu_{n}(E)=0$ when $Q_{n}(x) \neq 0$ on $E$. Let $M_{n}=\max _{x \in I}\left|Q_{n}(x)\right|$. Then, if

$$
\lim _{n \rightarrow \infty} M_{n}^{1 / n}=1 / 2
$$

it follows that

$$
\lim _{n \rightarrow \infty} \nu_{n}=\mu_{I} .
$$

Proof of Lemma 6.1. We have immediately from

the fact that

$$
\left|Q_{n}(x)\right| \leqq M_{n} \quad(x \in I)
$$

$$
\varlimsup_{n \rightarrow \infty}\left|Q_{n}(x)\right|^{1 / n} \leqq 1 / 2 \quad(x \in I),
$$

or

$$
\varliminf_{n \rightarrow \infty} U\left(v_{n}\right) \geqq \log 2 \quad(x \in I) .
$$

By Lemma 4.1 (a), in every increasing sequence of integers $\left(k_{n}\right)$, there is a subsequence $\left(t_{n}\right)$ such that $\left(\nu_{t_{n}}\right)$ converges, say with limit $\mu_{0}$. If we can show that $\mu_{0}=\mu_{I}$, using Lemma 4.1 (b) we find that (6.2) is established.

Now by Lemma 4.3

$$
U\left(x, \mu_{0}\right)=\lim _{n \rightarrow \infty} U\left(x, \nu_{l_{n}}\right)
$$

for $x \in I$, except for $x \in B$, a Borel set of type $F_{\sigma}$ and of capacity zero. Hence by (6.3)

$$
U\left(x, \mu_{0}\right) \geqq \log 2
$$


REGULAR BEHAVIOUR OF ORTHOGONAL POLYNOMIALS 145 for $x \in I \backslash B$. It thus follows from Theorem 1.3 that $\mu_{0}=\mu_{I}$, and the proof is complete.

We do not use the fact, nor do we prove it, but it is interesting to note that (6.1) is also necessary for (6.2) to hold.

LEMma 6.2 ([9] p. 178). Let $Q_{n}(x)$ be a polynomial of degree $n$ with

$$
M_{n}=\max _{x \in I}\left|Q_{n}(x)\right| \text {. }
$$

Then

$$
\left|Q_{n}^{\prime}(x)\right| \leqq n^{2} M_{n} \quad(x \in I)
$$

Proof of Theorem 1.10. We first note that, if (1.13) is satisfied for a measure $\mu \in \Omega$, then $c(\mu)=I$. Hence, by Theorem 1.8, there is a uniquely determined set of orthogonal polynomials $\left\{P_{n}(x \mid \mu)\right\}(n=0,1, \ldots)$. For a given integer $n>0$, let $\zeta_{n} \in I$ be a point when $\left|P_{n}(\zeta \mid \mu)\right|=M_{n}$. We will establish

$$
\left|P_{n}(y)\right| \geqq M_{n} / 2
$$

for $\left|y-\zeta_{n}\right| \leqq 1 /\left(2 n^{2}\right)(y \in R)$. By the mean value theorem for derivatives

$$
P_{n}(y)=P_{n}\left(\zeta_{n}\right)+P_{n}^{\prime}\left(x^{*}\right)\left(\zeta_{n}-y\right)
$$

for $x^{*}$ between $\zeta_{n}$ and $y$. Thus

$$
\begin{aligned}
\left|P_{n}(y)\right| & \geqq\left|P_{n}\left(\zeta_{n}\right)\right|-\left|P_{n}^{\prime}\left(x^{*}\right)\right|\left|\zeta_{n}-y\right| \\
& \geqq M_{n}-n^{2} M_{n}\left|1 /\left(2 n^{2}\right)\right|=M_{n} / 2,
\end{aligned}
$$

where we have used Lemma 6.2 in the second inequality.

We next consider the inequalities

$$
\begin{aligned}
(1 / 2)^{2(n-1)} & \geqq \int\left|T_{n}(x \mid I)\right|^{2} d \mu \geqq \int\left|P_{n}(x \mid \mu)\right|^{2} d \mu \\
& \geqq\left(M_{n}^{2} / 4\right) \int_{\zeta_{n}-1 /\left(2 n^{2}\right)}^{\zeta_{n}+1 /\left(2 n^{2}\right)} d \mu \geqq\left(M_{n}^{2} / 4\right) \omega\left(1 /\left(2 n^{2}\right)\right)
\end{aligned}
$$

We have used Lemma 3.5, (5.2), (6.5) and (1.14) successively in this chain of inequalities, yielding

We then obtain

$$
M_{n} \leqq \frac{(1 / 2)^{n-2}}{\omega\left(1 /\left(2 n^{2}\right)\right)^{1 / 2}}
$$

$$
\varlimsup_{n \rightarrow \infty} M_{n}^{1 / n} \leqq 1 / 2
$$

since

$$
\lim _{n \rightarrow \infty} \omega\left(1 /\left(2 n^{2}\right)\right)^{1 / 2 n}=\lim _{n \rightarrow \infty}\left(\omega\left(1 /\left(2 n^{2}\right)\right)^{1 / n}\right)^{1 / 2}=1
$$


by (1.13). Thus, by Lemma 6.1 ,

$$
\lim _{n \rightarrow \infty} \nu_{n}=\mu_{I}
$$

and so, by Definition 1.7 and the proof of Lemma 1.4, (1.1) is satisfied and $\mu$ is a regular measure.

Lemma 6.3. Let $p(x) \in P$, and define $p(x)=0$ in $\backslash I$. Let

$$
\tau(\delta)=\inf _{x \in I} \int_{x-\delta}^{x+\delta} p(x) d x .
$$

Then $p(x)$ is a regular weight function if

$$
\lim _{n \rightarrow \infty} \tau\left(1 /\left(2 n^{2}\right)\right)^{1 / n}=1 .
$$

Proof of Lemma 6.3. There are essentially no alterations from the proof of the previous lemma, and in (6.7) we use (6.8) and (6.9) instead of (1.14).

Proof of Theorem 1.9. We will next define an atomic measure $\mu \in \Omega$ which satisfies the condition

$$
\lim _{n \rightarrow \infty} \omega\left(1 /\left(2 n^{2}\right)\right)^{1 / n}=1,
$$

which by Theorem 1.10 is a sufficient condition for $\mu$ to be regular. Because (6.10) is satisfied, $c(\mu)=I$.

For each integer $n>0$, let $\mu_{n}$ be the measure determined by the conditions

$$
\mu_{n}\left(\left\{\frac{2 k-1}{2\left(n^{2}+1\right)}\right\}\right)=\lambda_{n} \quad\left(k=-n^{2},-n+1, \ldots, n^{2}+1\right),
$$

where $\lambda_{n}$ is a positive constant to be determined. Let $\mu=\sum_{n=1}^{\infty} \mu_{n}$. By [6] p. 232 this defines a measure, $c(\mu)=I$ and $\mu(I)=\sum_{n=1}^{\infty}\left(2 n^{2}+2\right) \lambda_{n}$. We choose

$$
\lambda_{n}=\frac{1}{\left(2 n^{2}+2\right) n(n+1)}
$$

so that $\mu(I)=1$.

We next note that, for any integer $n>0$,

since for $x \in I$

$$
\omega\left(1 /\left(2 n^{2}\right)\right) \geqq \lambda_{n},
$$

$$
\mu\left(\left(x-\frac{1}{2 n^{2}}, x+\frac{1}{2 n^{2}}\right)\right) \geqq \mu_{n}\left(\left(x-\frac{1}{2 n^{2}}, x+\frac{1}{2 n^{2}}\right)\right) \geqq \lambda_{n} .
$$

To see this, observe that, for $x \in I$, the interval $\left(x-\frac{1}{2} n^{-2}, x+\frac{1}{2} n^{-2}\right)$ has length $n^{-2}$, while the points $\left(k-\frac{1}{2}\right) /\left(n^{2}+1\right)\left(k=-n^{2},-n^{2}+1, \ldots, n^{2}+1\right)$ 
are spaced at a distance $1 /\left(n^{2}+1\right)$ apart, with the two extreme points at a distance $1 /\left(2\left(n^{2}+1\right)\right)$ from the end points of $I$. By combining (6.13) and (6.12), we see that $(6.10)$ holds.

Proof of Theorem 1.7. We associate with each integer $n>0$ a function $p_{n}(x)$ if the following way. First centre at each point $\left(k-\frac{1}{2}\right) /\left(n^{2}+1\right)$ $\left(k=-n^{2},-n^{2}+1, \ldots, n^{2}+1\right)$ an interval $l_{k, n}$ of length $1 / n^{2}\left(n^{2}+1\right)$. This choice ensures that one of the intervals $l_{k, n}$ lies entirely inside any interval of the form $\left[x-\frac{1}{2} n^{-2}, x+\frac{1}{2} n^{-2}\right]$. Define $p_{n}(x)$ to be $\lambda_{n} / \operatorname{meas}\left(l_{k, n} \cap E\right)$, where $\lambda_{n}$ is defined by (6.12) and the quantity in the denominator is not zero since $E \in N(I)$. We then note that $\int_{I} p_{n}(x) d x=\left(2 n^{2}+2\right) \lambda_{n}$, since the intervals $l_{k, n}$ are non-overlapping. Thus $p^{*}(x)=\sum_{1}^{\infty} p_{n}(x)$ is an integrable function by the monotone convergence theorem for integrals ([6] p. 84) and $\mathrm{S}\left(p^{*}(x)\right) \subset E$. Finally let $p(x)=p^{*}(x)+\chi_{E}(x)$, the added term being the characteristic function of $E$. Thus $\mathrm{S}(p(x))=E$. We will now show by Lemma 6.3 that $p(x)$ is a regular weight function. For any $x \in I$, we have

$$
\tau\left(\frac{1}{2} n^{-2}\right) \geqq \int_{x-\frac{1}{2} n^{-2}}^{x+\frac{1}{2} n^{-2}} p(x) d x \geqq \int_{x-\frac{1}{2} n^{-2}}^{x+\frac{1}{2} n^{-2}} p_{n}(x) d x \geqq \lambda_{n} .
$$

Thus, using (6.12), we see that

$$
\lim _{n \rightarrow \infty} \tau\left(1 /\left(2 n^{2}\right)\right)^{1 / n}=1,
$$

and so the proof is completed by reference to Lemma 6.3.

\section{Concluding remarks}

One question that we have dealt with is 'When is $p(x) \in P$ a regular weight function?' We have settled the question of when this fact can be ascertained from $\mathrm{S}(p(x))$ alone. Thus the next logical problem is to develop criteria for regularity in the case where $\mathrm{S}(p(x)) \in N(I)$ but is not a determining set. For each set $E \in N(I)$ that is not a determining set, we have seen that both regular and non-regular weight functions can occur having $E$ as support. We remark that the criterion (1.10) given in Theorem 1.8 distinguishes between weight functions that are very regular and those that are not on the basis of the values of $p(x)$.

Unlike the situation in the second-order theory, the question of characterizing regular measures, at least by our approach, is not settled by the characterization of regular weight functions.

The following two questions are related to each other, and to the result (1.11) for the second-order theory. 
148 REGULAR BEHAVIOUR OF ORTHOGONAL POLYNOMIALS

For $p(x) \in P$, does $\lim _{n \rightarrow \infty}\left(N_{n}(p)\right)^{1 / n}=1 / 2$ imply that the $p(x)$ is a regular weight function?

For $\mu \in \Omega, c(\mu)$ an infinite set, does $\lim _{n \rightarrow \infty}\left(N_{n}(\mu)\right)^{1 / n}=1 / 2$ imply that $\mu$ is a regular measure?

Our final question was raised by Paul Erdös in conversation. Let $O=\bigcup_{n=1}^{\infty} I_{n}$ be an open set dense in $I$, where $\left\{I_{n}\right\}$ are pairwise disjoint intervals. We have shown by our results that $\chi_{o}(x)$, the characteristic function of $O$, is a regular weight function if $\lim _{n \rightarrow \infty} C\left(\bigcup_{k=1}^{n} I_{k}\right)=1 / 2$. If $\lim _{n \rightarrow \infty} C\left(\bigcup_{k=1}^{n} I_{k}\right)<1 / 2$, is $\chi_{o}(x)$ necessarily not a regular weight function? The techniques of Lemma 1.3 (c) show that $\chi_{0}(x)$ is not a regular weight function if, in addition, the length of $I_{n}$ tends to zero sufficiently rapidly.

We finally remark that [10] and [11] contain the preliminary form of several techniques used in this paper, as well as related results.

\section{REFERENCES}

1. P. Erdös and P. Turan, 'On interpolation. III', Ann. of Math. 41 (1940) 510-55.

2. L. Ya. Gerontmous, Orthogonal polynomials (Consultants Bureau, New York, 1961).

3. M. HeIns, Selected topics in the classical theory of functions of a complex variable (Holt, Rinehart, and Winston, New York, 1962).

4. E. Hrwle, Analytic function theory (Ginn and Company, New York, 1962).

5. Ch. J. DE LA VALlée Poussin, Le potentiel logarithmique (Gauthier-Villars, Paris, 1949).

6. H. L. Royden, Real analysis, 2nd edn (Macmillan, New York, 1968).

7. J. Shohat and J. TAMarkin, The problem of moments, Mathematical Surveys, No. 1 (American Mathematical Society, New York, 1943).

8. G. SzEGö, Orthogonal polynomials, Colloquium Publication XXIII (American Mathematical Society, New York, 1939).

9. M. TsuJr, Potential theory in modern function theory (Maruzen, Tokyo, 1959).

10. J. L. UllmaN, 'A criterion for arcsine distribution', J. Math. Mech. 10 (1967) $1168-76$.

11. 'On weighted Tchebycheff polynomials', Orthogonal expansions and their continuous analogues (S. Illinois University Press (1968) 51-53).

12. H. WIDOM, 'Extremal polynomials associated with a system of curves in the complex plane', Advances in Math. 3 (1969) 127-232.

\section{University of Michigan \\ Ann Arbor, Michigan}

